\title{
The Limit Load Calculations for Pipelines with Axial Complex-Shaped Defects
}

\author{
A. V. Bogdan, ${ }^{a}$ I. V. Lokhman, ${ }^{\text {b }}$ S. M. Ageev, ${ }^{a}$ and I. V. Orynyak ${ }^{a}$ \\ ${ }^{a}$ Pisarenko Institute of Problems of Strength, National Academy of Sciences of Ukraine, \\ Kiev, Ukraine \\ ${ }^{\text {b }}$ SC Ukrtransgas, Kiev, Ukraine \\ Based on the analytical model for the plastic limit state, a numerical procedure for estimating the \\ remaining strength of a complex-shaped defect has been developed. Comparison of the calculation \\ results obtained by the proposed procedure with the experimental data and the calculation results \\ obtained by other methods showed its efficiency.
}

Keywords: defect, pipe, strength reduction factor, internal pressure, ultimate strength, limit load.

Introduction. An important constituent of the pipeline reliability assurance is the in-line inspection, using which the remaining wall thickness $t_{n}$ of a pipe at its discrete points is found. Each of the points is characterized by the axial coordinate along the pipeline $s$ and the angular coordinate $\varphi$ around the circle of the cross-section.

For further calculations the two-dimensional system is reduced to a unidimensional one: the maximum defect depth is projected onto the axial line as specified in DNV-RP-F101 [1]. An example of this system of points, which is called a complex defect, is presented in Fig. 1. The simplest method for analyzing the strength of a complex defect is its replacement by a rectangular defect whose length is equal to that of the complex defect $l$ and depth is equal to the maximum depth of the complex defect $d$. Clearly, the results obtained in this way would be rather conservative. However, they are useful in the case where there is a need to obtain, within a short period of time, an approximate estimate of the complex defect strength and to get an answer to whether it is necessary at all to carry out a more accurate calculation that takes into account the real profile of a complex defect. Thus, we initially consider some approaches to strength estimation for defects of typical shapes (rectangular, elliptic, and parabolic) and then the calculation methods that involve the use of a real profile of a complex defect.

\section{Estimation of Strength of a Pipe with Defects of Typical Shape.}

1.1. Treatment of an Isolated Defect According to DNV-RP-F101. One of the current and generally recognized techniques for estimating corrosion defects is the international standard DNV Recommended Practice [1]. According to this practice, for an isolated defect of length $l$ and depth $d$, the expression for determining the internal failure pressure is rather simple and has the following form:

$$
\left.P=\left(2 t \sigma_{u}\left(1-\frac{d}{t}\right)\right) /(D-t)\left(1-\frac{d}{t Q}\right)\right),
$$

(C) A. V. BOGDAN, I. V. LOKHMAN, S. M. AGEEV, I. V. ORYNYAK, 2009 ISSN 0556-17IX. Проблемы прочности, 2009, № 1 
where

$$
Q=\sqrt{1+0.31(l / \sqrt{D t})^{2}}
$$

$\sigma_{u}$ is the ultimate strength of the pipe steel, $t$ is the pipe wall thickness, and $D$ is its outer diameter. According to [1], relationship (1) is empirical and derived on the basis of a great number of calculations by the finite element method (FEM) and full-scale experiments. In contrast to this approach to the estimation of the remaining strength of a pipe with single defects, we consider below an analytical model which has been developed by us and is based on the limit state theory.

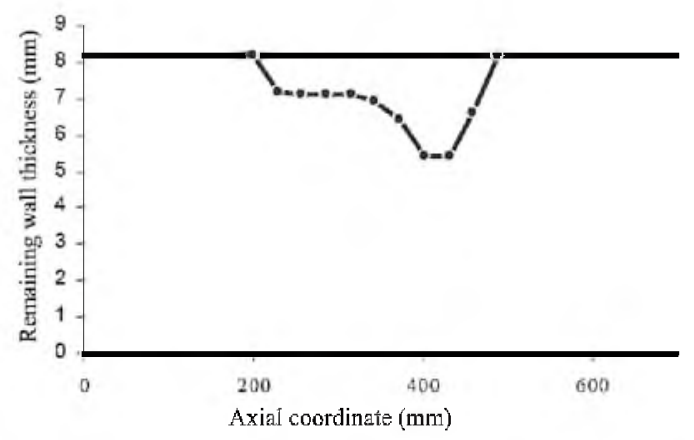

Fig. 1. Profile of a complex defect.

1.2. The Analytical Model. The strength condition for a defected pipe can be written as

$$
p \leq \alpha(\lambda, \tau) \frac{\sigma_{u} t}{R}
$$

where $\alpha(\lambda, \tau)$ is the strength reduction factor, $\lambda=l / \sqrt{R t}, R$ is the inner radius, $t$ is the wall thickness, $\tau=t_{n} / t, t_{n}$ is the remaining wall thickness, $t_{n}=t-d$, $\sigma_{u}$ is the ultimate strength of the pipe material, and $p$ is the applied internal pressure. We shall consider the basic idea of the $\alpha(\lambda, \tau)$ determination.

Consider the equilibrium equation in the radial direction:

$$
p=\frac{N_{\varphi}}{R}+\frac{d Q_{x}}{d x},
$$

where $N_{\varphi}$ is the circumferential force, $\varphi$ is the circumferential angular coordinate, $x$ is the axial coordinate, and $Q_{\varphi}$ and $Q_{x}$ are the transverse forces. For a defect-free pipe, each point of the pipe is under identical conditions, therefore, the transverse forces are equal to zero. Since $N_{\varphi}=\sigma_{\varphi}$, the limit state is attained when $\sigma_{\varphi}=\sigma_{u}$, therefore, the admissible internal pressure $p_{L L}(\tau=1)=\sigma_{u} t / R$ or $\alpha(\tau=1)=1$.

For a section with a part-through defect of the maximum depth $d$, the maximum value of $N_{\varphi} / R$ in (4) is equal to $\sigma_{u}(t-d) / R$ and this force cannot resist the larger values of the internal pressure $p$. Thus, the presence of an axial defect results in the imbalance between the circumferential stresses and the 
internal pressure which has to be counterbalanced by an increment in the transverse forces to maintain equilibrium. The transverse forces, in turn, induce bending moments. The state of the cylinder changes to the limit state when the bending moments reach the critical values that meet the chosen criterial conditions. The above considerations are presented in more detail in [2], where the following expressions for $\alpha_{L L}$ were found:

$$
\alpha_{L L}=\frac{1+2 \lambda^{2} \tau_{0}\left(1-\tau_{0}\right)}{1+2 \lambda^{2}\left(1-\tau_{0}\right)}
$$

for a rectangular defect,

$$
\alpha_{L L}=\frac{1+\lambda^{2}\left(1-\left(1-\tau_{0}\right) \pi^{2} 3 / 32\right)\left(1-\tau_{0}\right) 4 / 3}{1+\lambda^{2}\left(1-\tau_{0}\right) 4 / 3}
$$

for a semi-elliptical defect, and

$$
\alpha_{L L}=\frac{1+\lambda^{2}\left(1-\left(1-\tau_{0}\right) 8 / 9\left(1-\tau_{0}\right)\right.}{1+\lambda^{2}\left(1-\tau_{0}\right)}
$$

for a parabolic defect.

As was noted above, the replacement of a complex defect by a rectangular one leads to rather conservative estimates. Therefore, below we consider the approaches which take into account the real shape of a complex defect and yield a more accurate estimate of the strength.

\section{Strength Calculation for Complex Defects.}

2.1. The Effective Area for a Complex Defect. One of the simplest methods of strength calculation for a pipeline with a complex defect involving the use of its real profile is the method based on the concept of the effective area.

In [3], the following expression for strength evaluation for a complex defect is proposed:

$$
\sigma_{f}=\bar{\sigma}\left(1-\frac{A}{A_{0}}\right) /\left(1-\frac{A}{A_{0}} M^{-1}\right),
$$

where $A$ is the effective area, $A_{0}$ is the total area across the wall thickness in the longitudinal direction, $M$ is the Folias factor, and $\bar{\sigma}$ is the yield strength of the pipeline material. With this approach, the multiplier $\left(1-A / A_{0}\right)$ can be interpreted as the ratio of the average remaining wall thickness $t_{n a}$ to the original defect-free wall thickness $t$ of the defected area. Different zones of the complex defect are examined sequentially and the minimum wall thickness is chosen. The subjective nature of this model is obvious.

Another currently available method of strength analysis for a complex defect is the method proposed in DNV-RP-F101 [1]. The basic idea of this method consists in splitting a complex defect into simple ones with their strength calculation using the expression described in item 1.1. 
As an alternative to the existing traditional approaches, the researchers of the Pisarenko Institute of Problems of Strength developed a numerical procedure for assessing complex shaped defects, which rests upon clear-cut physical models.

2.2. Numerical Procedure for Determining the Strength Reduction Factor for a Complex-Shaped Defect. Let us introduce the notion of the dimensionless internal pressure $\bar{p}=(p R) /\left(\sigma_{u} t\right)$, where $\sigma_{u}$ is the ultimate strength of the pipeline material. We mark $N$ points on the profile of a complex defect at the interval $d x=t$, where $t$ is the wall thickness. Each point is characterized by the remaining strength $\sigma_{i}=\left(t_{n i} / t\right) \sigma_{u}$, where $t_{n i}$ is the remaining wall thickness at the point numbered $i$.

The basis of the critical state model is that the increments in the applied moment to the left of the point under consideration (towards smaller point numbers) are positive and those to its right, are negative. In addition, we assume that when moving to the left, we have negative increments $d x=-t$. These assumptions were made for the purpose of similarity of further computations.

We assign a numerical value to the dimensionless internal pressure $\bar{p}$. Keeping in mind the above considerations, we search for points where the strength condition may be not satisfied. Before formulating the procedure of searching, let us find the value of the increment in the transverse force on the segment $d x$ (the subscript $i$ on the remaining strength $\sigma$ is omitted):

$$
\frac{d Q_{x}}{d x}=p-\frac{N}{R}=\frac{\bar{p} \sigma_{u} t}{R}-\frac{\sigma t}{R}=\frac{\sigma_{u} t}{R}[\bar{p}-\bar{\sigma}],
$$

where $\bar{\sigma}=\sigma / \sigma_{u}$ is the dimensionless remaining strength.

It is clear that according to the assumption of a positive increment in the bending moment to the left of the point with the number $k$, it is necessary that the condition of the transverse force $Q_{x}$ decrease be fulfilled. Thus

$$
\bar{p}-\sigma_{k} \geq 0
$$

because $d x<0$. Condition (8) is a necessary condition to choose a point of possible failure.

Let us assume that on passing sequentially the complex defect profile points, the point satisfying condition (8) is found. Then we take $k=0$ for this point and consider that the points with negative numbers are to the left of this point and those with positive numbers to the right of it. Also assume that the transverse force $Q_{x}=0$ at this point. The aim of the calculation is to find the increment in the bending moment and to compare it with its critical value. So we move to the left (in the direction of the negative numbers of the points). The transverse forces can be found from the following expressions:

$$
\begin{aligned}
& Q_{-1}=Q_{0}-\frac{\sigma_{a} t}{R}\left(\bar{p}-\sigma_{0}\right) d x, \\
& \cdots \cdots \cdots \cdots \cdots \cdots \cdots \cdots \cdots \cdots \cdots \cdots \cdots \cdots \cdots \cdots \cdots \cdots \cdots \cdots \cdots \cdots \\
& Q_{-i}=Q_{-i+1}-\frac{\sigma_{a} t}{R}\left(\bar{p}-\sigma_{-i+1}\right) d x,
\end{aligned}
$$


or in dimensionless quantities:

$$
\begin{aligned}
& \bar{q}_{-1}=\bar{q}_{0}+\left(\bar{p}-\sigma_{0}\right), \\
& \cdots \cdots \cdots \cdots \cdots \cdots \cdots \cdots \cdots \cdots \cdots \cdots \cdots \cdots \cdots \cdots \cdots \cdots \cdots \cdots \\
& \bar{q}_{-i}=\bar{q}_{-i+1}+\left(\bar{p}-\sigma_{-i+1}\right),
\end{aligned}
$$

where $Q_{-i}=\bar{q}_{-i} \frac{\sigma_{a} t^{3}}{R}$.

Let us find the increments in the bending moments:

$$
\begin{aligned}
& M_{0}=Q_{0} t+\frac{\sigma_{a} t}{R}\left(p-\sigma_{0}\right) \frac{t^{2}}{2} \\
& M_{-i}=M_{-i+1}+Q_{-i+1}+\frac{\sigma_{a} t}{R}\left(p-\sigma_{-i+1}\right) \frac{t^{2}}{2}
\end{aligned}
$$

or using (10) and (11) in dimensionless quantities

$$
\begin{aligned}
& \bar{m}_{0}=\frac{\bar{p}-\sigma_{0}}{2}, \\
& \ldots \ldots \ldots \ldots \ldots \ldots \ldots \ldots \ldots \ldots \ldots \ldots \ldots \ldots \ldots \ldots \ldots \ldots \\
& \bar{m}_{-i}=\bar{m}_{-i+1}+\bar{q}_{-i+1}+\frac{\left(\bar{p}-\sigma_{-i+1}\right)}{2},
\end{aligned}
$$

where $M_{-i}=\bar{m}_{-i} \frac{\sigma_{a} t^{3}}{R}$.

The condition of strength has the form $M_{-i} \leq \frac{\sigma_{a} t^{2}}{4}$ or in a dimensionless form

$$
\bar{m}_{-i} \leq \frac{R}{4 t}
$$

We keep on moving to the left until $\bar{m}_{-i} \geq 0$ and $\bar{m}_{-i} \leq \frac{R}{4 t}$. If strength condition (13) is not fulfilled, we go to the right (towards positive subscripts). Otherwise, we search for the next point where the condition of fracture possibility (8) holds true.

We move to the right in a similar way, with the only difference that on the right, according to the model assumption, $\bar{m}_{i}$ should be negative. If on the right the strength condition (13) is not fulfilled either, fracture occurs. If no point where fracture occurs is found for a given dimensionless internal pressure, we increase the value of the dimensionless internal pressure and repeat the above steps of the numerical procedure. If such a point has been found, we decrease the value of the internal pressure. The search technique can be similar, e.g., to that of 
dividing a segment in two. In this case, the dimensionless internal pressure will be determined with a prescribed accuracy. It is obvious that the obtained value of the dimensionless internal pressure $\bar{p}$ will be equal to the strength reduction factor $\alpha$.

The validity of the developed algorithm was checked on two model examples, wherein the geometrical parameters of defects were set in conventional units related to a single step $d x=t=1$.

Example 1. Consider a rectangular defect with the following parameters: length $L=5, R / t=20$, and $t_{n} / t=0.3$. Calculations by formula (5a) gives $\alpha=0.5545$ and by the algorithm constructed $\alpha=0.556$.

Example 2. Consider two rectangular defects of length $2 L$ and depth $d$ in a pipe, the distance between the defects being equal to $2 k$ (Fig. 2).

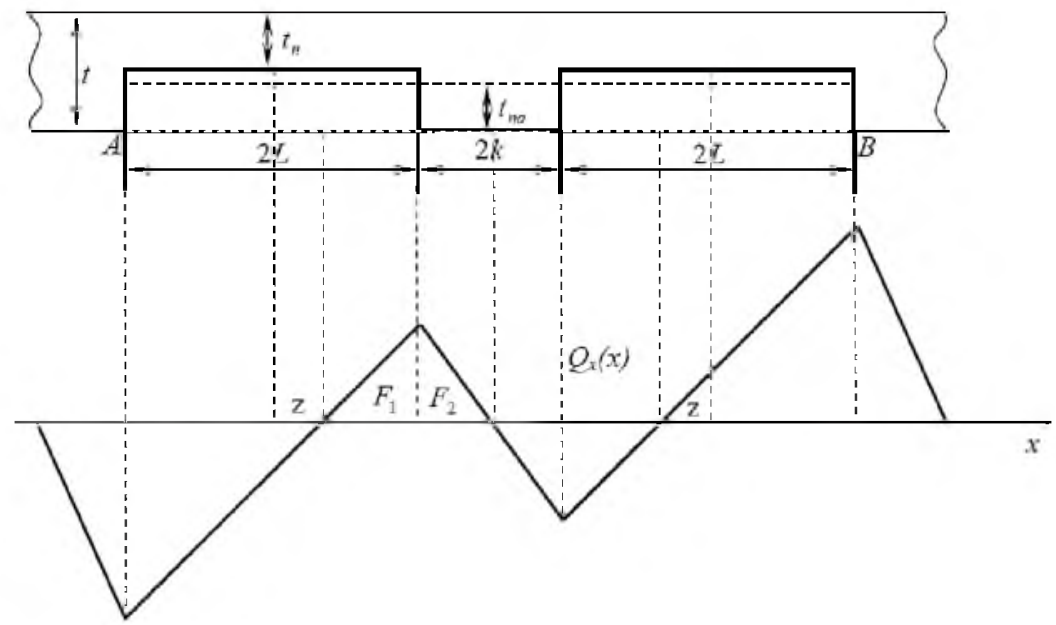

Fig. 2. Stressed state for two symmetrical defects.

The value of the strength reduction factor $\alpha$ is numerically found from a system of equations where the first equation can be obtained from expression (5a) assuming that the defect half length is equal to $L+z(z$ is the displacement of the point, where $Q_{x}=0$ with respect to the defect center) and the second equation is obtained from the conditions of equality of the areas of figures $F_{1}$ and $F_{2}$ :

$$
\left\{\begin{array}{l}
\alpha=\left(1+2 \frac{(L+z)^{2}}{R t} \tau(1-\tau)\right) /\left(1+2 \frac{(L+z)^{2}}{R t}(1-\tau)\right), \\
(l-z)(\alpha-\tau)=k(1-\alpha) .
\end{array}\right.
$$

Consider two rectangular defects (Fig. 2) with the following parameters: $L=9, R / t=80$, and $t=1$, i.e. $\lambda=1.0125$ and $t_{n} / t=0.3$. We calculate the strength of such a defective segment by the developed semi-analytical procedure for two defects with the use of the proposed universal numerical algorithm and a technique described in DNV-RP-F101 [1].

Figure 3 presents the dependences of the strength reduction factor $\alpha$ on the dimensionless distance between the defects $k / L$ obtained by the method based on relations (14), with the use of the numerical procedure, and the procedure 
described in the DNV-RP-F101 [1] (dashed line). The numerical and analytical solutions (14) coincide and are presented by a solid line.

Examples 1 and 2 indicate that the numerical algorithm for determining the strength reduction factor is correct and devoid of the drawbacks associated with simplification or schematization, and it is appropriate for strength calculation for a pipe with an arbitrary strength distribution at each point. Further we consider examples of strength calculation for real complex-shaped defects using different computation methods.

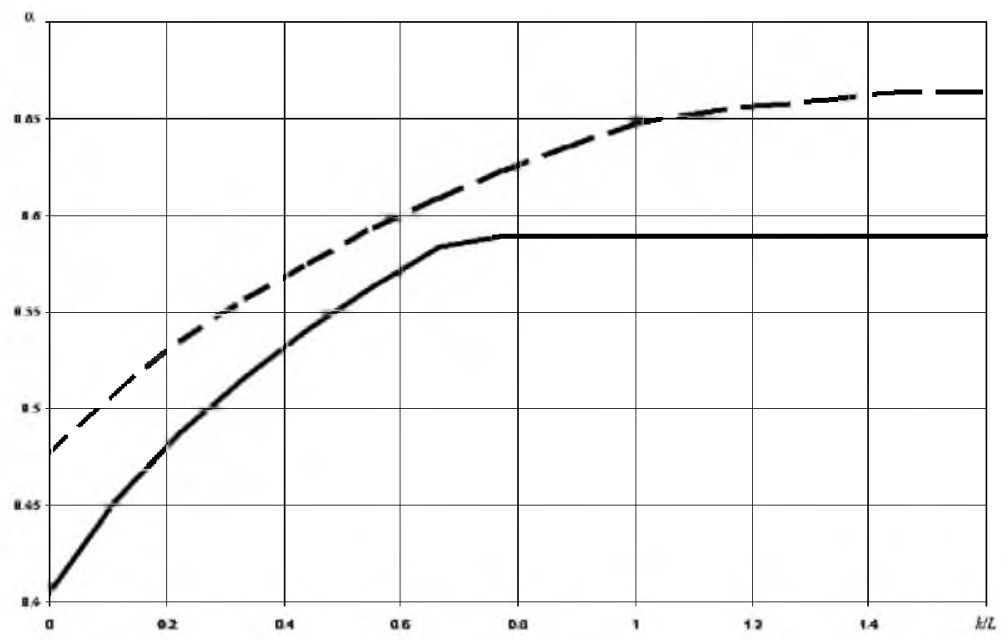

Fig. 3. Strength reduction factor $\alpha$ versus dimensionless distance between the defects $k / L$ : solid line - numerical procedure, dashed line - DNV-RP-F101 [1].

2.3. Determination of Strength for Real Defects. Consider determination of the internal failure pressure for real defects.

Example 1. (DNV-RP-F101 [1], B.3.2). The input parameters of a model defect: $D=611 \mathrm{~mm}, t=8.2 \mathrm{~mm}$, and $f_{u}=571 \mathrm{~N} / \mathrm{mm}^{2}$. The profile geometry for a complex shaped defect is presented in Table 1 .

$\mathrm{T}$ a b 1 e 1

The Profile Geometry for a Complex Shaped Defect

\begin{tabular}{|c|c|c|c|c|c|}
\hline Point number & Length $(\mathrm{mm})$ & Depth $(\mathrm{mm})$ & Point number & Length $(\mathrm{mm})$ & Depth $(\mathrm{mm})$ \\
\hline 1 & 0 & 0 & 7 & 173.4 & 1.8 \\
\hline 2 & 28.9 & 1.0 & 8 & 202.3 & 2.8 \\
\hline 3 & 57.8 & 1.1 & 9 & 231.2 & 2.8 \\
\hline 4 & 86.7 & 1.1 & 10 & 260.1 & 1.6 \\
\hline 5 & 115.6 & 1.1 & 11 & 289.0 & 0 \\
\hline 6 & 144.5 & 1.3 & & & \\
\hline
\end{tabular}

Strength calculations for such a defect are performed using computer programs based on the algorithm from DNV-RP-F101 [1] and the numerical procedure developed by us. 
Results of calculations:

- failure pressure calculated according to the DNV: $P_{f}=13.22 \mathrm{~N} / \mathrm{mm}^{2}$;

- failure pressure calculated by our numerical procedure: $P_{f}=13.34 \mathrm{~N} / \mathrm{mm}^{2}$.

Example 2 ([4]). The input parameters of a real defect (its profile geometry is shown in Fig. 4): $D=457.7 \mathrm{~mm}, t=6.04 \mathrm{~mm}$, and $f_{u}=428.5 \mathrm{~N} / \mathrm{mm}^{2}$.

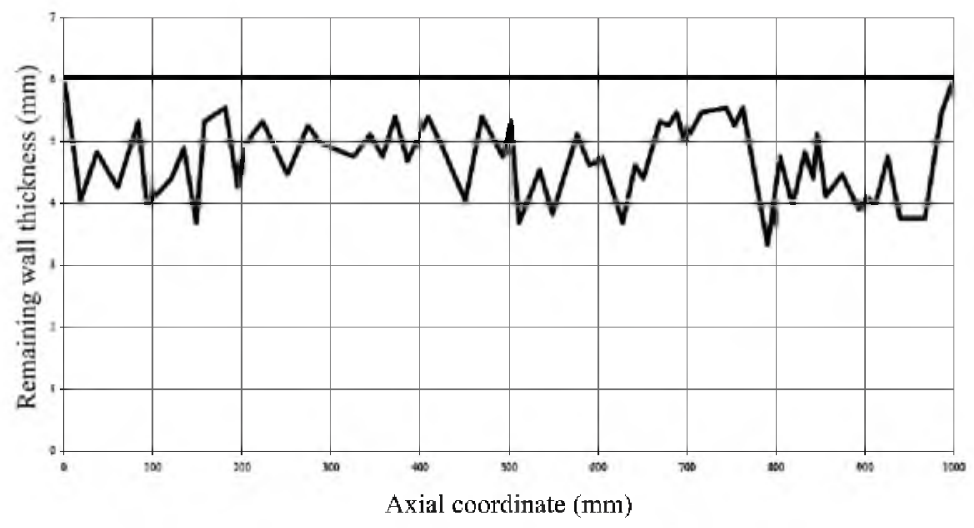

Fig. 4. Profile of a complex-shaped defect [3].

Results of strength calculation:

- failure pressure calculated according to the DNV: $P_{f}=8.51 \mathrm{~N} / \mathrm{mm}^{2}$;

- failure pressure calculated using the effective area: $P_{f}=8.70 \mathrm{~N} / \mathrm{mm}^{2}$;

- failure pressure calculated by the numerical procedure from item 2.2: $P_{f}=8.71 \mathrm{~N} / \mathrm{mm}^{2}$;

- experimental failure pressure: $P_{f}=10.13 \mathrm{~N} / \mathrm{mm}^{2}$.

The results obtained demonstrate that the numerical procedure for calculating the failure pressure developed by us is correct, physically justified and enables obtaining more accurate estimates of the failure pressure in a pipe as compared with the known calculation procedures.

1. DNV Recommended Practice - DNV-RP-F101 - Corroded Pipelines, Det Norske Veritas, Norway (2004).

2. I. V. Orynyak, "Leak and break models of ductile fracture of pressurized pipe with axial defects," in: Proc. IPC2006 6th Int. Pipeline Conf. (Sept. 25-29), Calgary, Canada (2006).

3. J. F. Kiefner and P. H. Vieth, "PC program speeds new criterion for evaluating corroded pipe," Oil \& Gas J., 88, No. 34, 91-93 (1990).

4. R. D. Souza, A. C. Benjamin, R. D. Vieira, et al., "Part 4: Rupture tests of pipeline segments containing real long corrosion defects," Exp. Tech., 31, No. 1, 46-51 (2007). 